\title{
Intensity and Uniformity Enhancement of Dielectric Barrier Discharge by Using Silver-Coated Electrode
}

\author{
Received 9 September, 2020; revised 12 November, 2020; accepted 19 November, 2020
}

\author{
Sang-Min Lee ${ }^{\mathrm{a}}$ and Jun Choi $\mathrm{i}^{\mathrm{b}, *}$ \\ aSchool of Electrical Engineering, University of Ulsan, Ulsan 44610, Republic of Korea \\ bAdvanced Forming Process R\&D Group, Korea Institute of Industrial Technology, Ulsan 44413, Republic of Korea
}

*Corresponding author E-mail: junchoi@kitech.re.kr

\begin{abstract}
The intensity and uniformity of the dielectric barrier discharge (DBD) were studied via fabrication. The DBD can be achieved by various methods using dielectrics and electrodes; however, depending on the device configuration and fabrication method, unnecessary air gaps may occur between the dielectric and electrode. The air gap increases the breakdown voltage for plasma generation, thereby affecting the characteristics of the DBD. In this study, an inner electrode was formed on the surface of a dielectric by the silver mirror reaction to eliminate the air gap; in this technique, silver is deposited on the dielectric surface by a reducing organic compound. The DBD images of the silver-coated inner electrode were investigated using an intensified charge coupled device (ICCD) camera and the OpenCV library of Python for quantitatively evaluating performance measures such as intensity and uniformity. In addition, the reactive oxygen species (ROS) generated by DBD were measured with a band-pass filter and the ICCD camera. When the DBD device was driven by same voltages, the silver-coated inner electrode showed improved intensities and uniformities of up to 7.98 and 1.75 times, respectively. Moreover, the ROS intensities and uniformities were improved by up to 57.8 and 9.27 times, respectively.
\end{abstract}

Keywords: Dielectric barrier discharge, Image processing, Intensity, Silver mirror reaction, Uniformity

\section{Introduction}

The dielectric barrier discharge (DBD) process has been widely used in applications such as inactivation of bacteria, air purification, biomedicine, surface treatments, blood coagulation and fruit storage [1-5]. The DBD device has a simple structure comprising two electrodes and a dielectric, and it can be manufactured by various methods. However, complex forms of the DBD device, such as a cylindrical form, may have unnecessary air gaps between the electrode and dielectric. As this air gap increases, the electric field weakens, and the breakdown voltage for plasma generation increases [6]. Therefore, a fabrication method that avoids unnecessary air gaps is worth investigating to improve the characteristics of the DBD.

Qizheng et al. studied the uniformity of the DBD based on the shape of the mesh electrode and used image processing methods to evaluate the uniformity of the DBD [7]. However, they did not explain the uniformity of the reactive oxygen species (ROS) generated by the $\mathrm{DBD}$. The ROS generated by the DBD can achieve bacterial inactivation. Ma et al. supposed that the cell membrane could be oxidized by the ROS, which may damage the protein and nucleic acids inside the cells to cause bacteria to become inactive [8]. Thus, generation of ROS is important for sterilizing bacteria using DBD devices.

In this paper, we propose a fabrication method for the inner electrode to reduce the formation of unnecessary air gaps. To eliminate the air gap between the electrode and dielectric, the inner electrode was coated on the surface of the dielectric by a silver mirror reaction. The inner electrode was either coated or not coated to analyze the intensity and uniformity of electron density improvements. The DBD im- ages were observed using an intensified charge coupled device (ICCD) camera and processed with the OpenCV library of Python to quantitatively evaluate performance measures for the intensity and uniformity. Furthermore, the ROS produced by the DBD was observed using a band-pass filter along with the ICCD camera; the intensity and uniformity of the ROS were thus evaluated based on the method used for fabrication of the inner electrode.

\section{Experimental details}

\subsection{DBD device fabrication}

The DBD device is designed in a cylindrical form and consists of two electrodes and a dielectric. As a common component, the outer electrode is a stainless mesh of $0.5-\mathrm{mm}$ thickness and $0.6-\mathrm{mm}$ aperture length $(L)$, and the dielectric is a quartz tube of $1.5-\mathrm{mm}$ thickness, $30-\mathrm{mm}$ diameter, and $165-\mathrm{mm}$ length. Because the outer electrode is a stainless mesh of $0.6-\mathrm{mm}$ aperture length $(L)$, we presumed $n_{e}=4.63 \times 10^{3} \mathrm{~cm}^{-3}$. The $\mathrm{L}$ can take the place of the seed electron number density $\left(n_{e}\right)$ in the manner of $L^{3}=1 / n_{e}$ [7]. Figure 1 shows the inner electrode of the DBD device that is manufactured using a copper plate of 0.1 -mm thickness and 165 -mm length without and with silver coating. Figure 1(b) shows the inner electrode of the DBD device that is coated with silver using the silver mirror reaction method. The silver mirror reaction involves mixing silver nitrate solution (AgNO3) with ammonia and a reducing organic compound to extract silver, which forms a mirror-like surface $[9,10]$. Air gaps between the quartz tube and mesh do not exist. Moreover, the silver- 

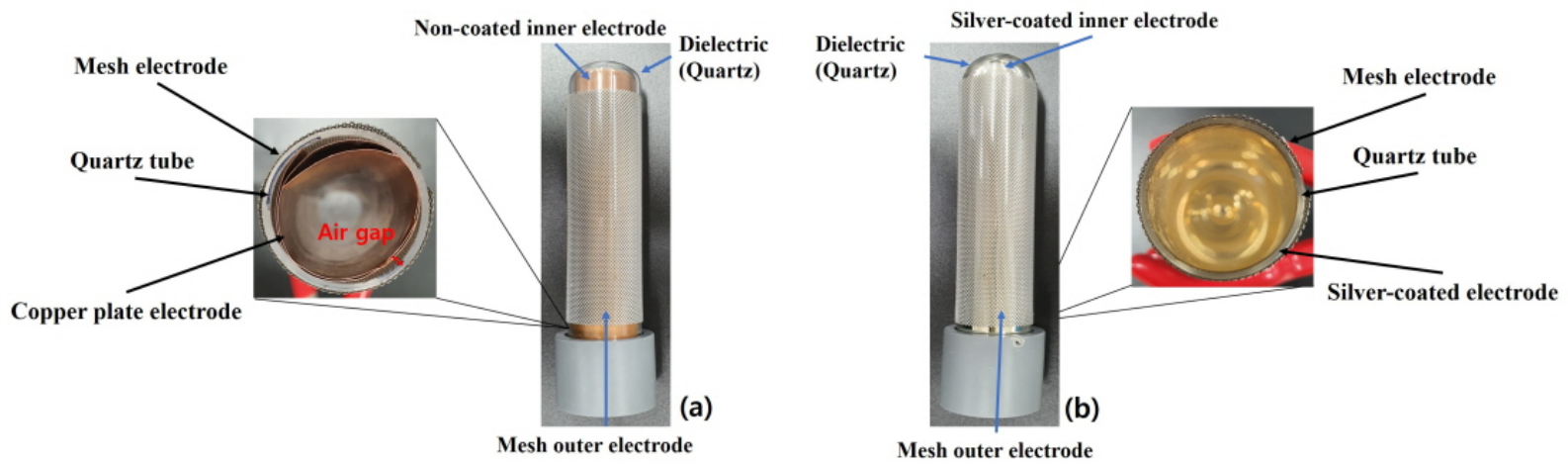

Figure 1. Structure of dielectric barrier discharge (DBD) devices using quartz and a mesh outer electrode with the (a) non-coated inner electrode and (b) silver-coated inner electrode.

coated inner electrode can be manufactured without causing any unnecessary air gaps as shown in Fig. 1(b). However, the non-coated inner electrode using the copper plate can have air gaps of up to 1.5 $\mathrm{mm}$ as shown in Figure 1(a).

\subsection{Experimental setup}

Figure 2 shows the schematic of the experimental setup to obtain the DBD images with an ICCD camera. A sinusoidal voltage in the range of 6 to $11 \mathrm{kV}$ (peak-to-peak) with a frequency of $3 \mathrm{kHz}$ was driven by the DBD device. The applied voltage was measured using an oscilloscope (Keysight DSOX6004A) and a high-voltage probe (Keysight N2891A, 1000:1). The corresponding current was measured using a current probe (Keysight N7026A). The DBD images were observed using the ICCD camera (Andor iStar) according to the applied voltage. The ICCD camera has a wider wavelength range of operation than visible light, i.e., $200-900 \mathrm{~nm}$. The $\mathrm{OH}$ radical is a type of ROS having an emission wavelength of $309 \mathrm{~nm}$, and NO radicals are ROS with emission wavelengths of 204-280 nm [11, 12]. The wavelength ranges were obtained using optical filters (Andover) so that the $\mathrm{OH}$ radical could be observed with the ICCD camera. The optical filter used here was a band-pass filter centered at $308.78 \mathrm{~nm}$ with a bandwidth of $11.20 \mathrm{~nm}$. The optical density of the band-pass filter was 4 on average from X-ray to FIR.

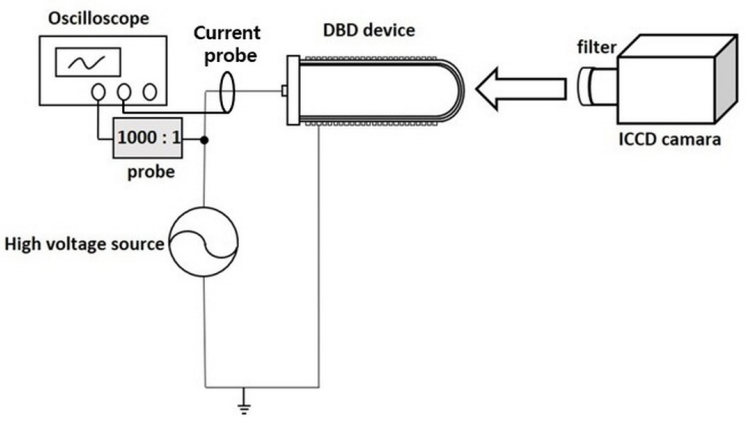

Figure 2. Schematic of experimental setup for observing DBD using the intensified charge coupled device (ICCD) camera. The DBD device is driven by a high voltage in the range of $6 \mathrm{kV}$ to $11 \mathrm{kV}$, which is measured using an oscilloscope with a 1000:1 probe. The corresponding current is measured using a current probe.
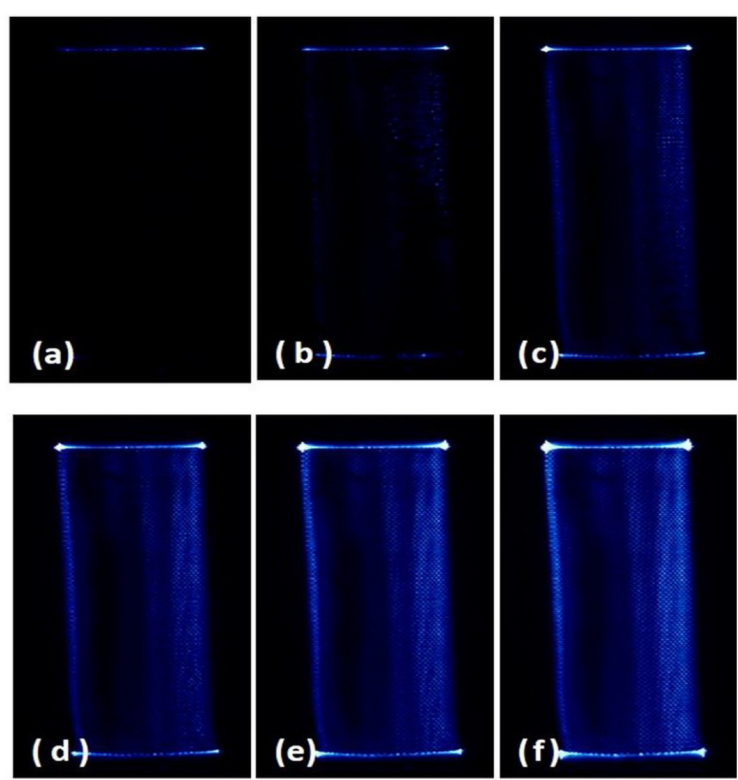

Figure 3. DBD images obtained using the silver-coated inner electrode applied with voltages of (a) 6 , (b) 7, (c) 8, (d) 9, (e) 10, and (f) $11 \mathrm{kV}$ are observed with the ICCD camera without any filters.

\section{Results and discussion}

\subsection{Comparison of intensities and uniformities for the two types of electrodes}

The DBD images were first observed using the ICCD camera without any filters. Figures 3 and 4 show the DBD images obtained according to the applied voltages; the DBD areas and intensities increase with increases in the applied voltages. From a visual perspective, for a given applied voltage, the intensities of the silver-coated inner electrode are greater than that of the non-coated inner electrode. The intensity of the non-coated inner electrode for an applied voltage of $11 \mathrm{kV}$ is similar to that of the silver-coated inner electrode for an applied voltage of $8 \mathrm{kV}$. As a result, the silver-coated inner electrode is seen to produce an increased electric field owing to the reduced distance between the electrode and dielectric.

Image processing techniques were used to quantitatively compare the intensities and uniformities of DBD for the applied voltage. The DBD images obtained from the ICCD camera are converted to grayscale images using the OpenCV library of Python. The grayscale images can be represented by two-dimensional matrices of size $M \times$ $\mathrm{N}$, where each pixel has the intensity information as $\mathrm{g}(\mathrm{x}, \mathrm{y})$. The $\mathrm{X}$-axis 

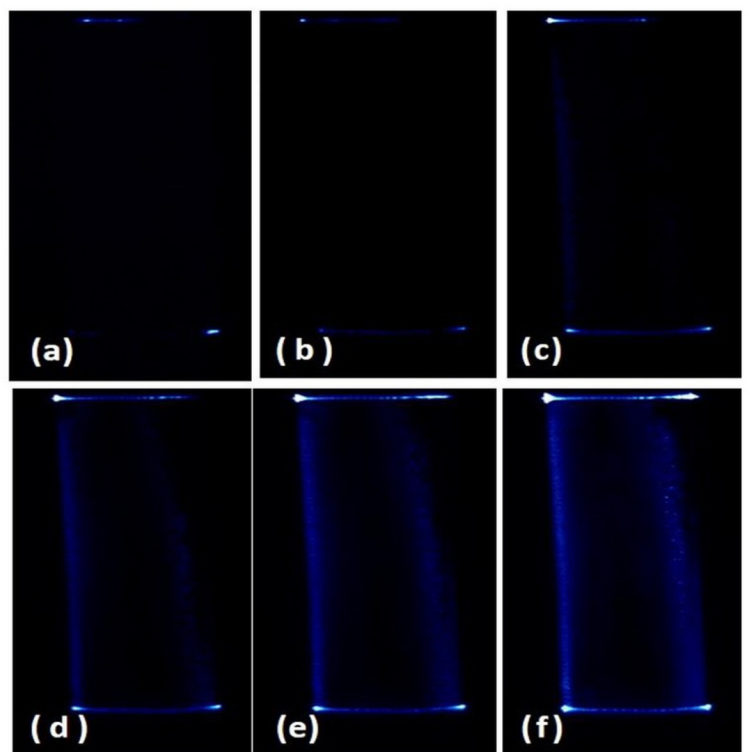

Figure 4. DBD images obtained using the non-coated inner electrode applied with voltages of (a) 6, (b) 7, (c) 8, (d) 9, (e) 10, and (f) $11 \mathrm{kV}$ are observed with the ICCD camera without any filters.

and $\mathrm{Y}$-axis of the histogram represent the pixel intensity and number of pixels, respectively. If a pixel has high intensity, its value would be close to 255; otherwise, its intensity would be close to zero. Therefore, the intensity and uniformity of the DBD can be obtained from the histogram.

\section{Average intensity}

The average intensity is related to the average graylevel value obtained from the histogram. The average graylevel for an $\mathrm{M} \times \mathrm{N}$ image can be determined as follows:

$$
\bar{g}=\frac{1}{M \times N} \sum_{x=0}^{M-1} \sum_{y=0}^{N-1} g(x, y)
$$

Figure 5 shows the average intensities depending on the applied voltage as obtained using Eq. (1); the silver-coated inner electrode has a higher average intensity than the non-coated inner electrode for applied voltages in the range of 6 to $11 \mathrm{kV}$. In addition, the average intensity of the non-coated inner electrode for an applied voltage of 11

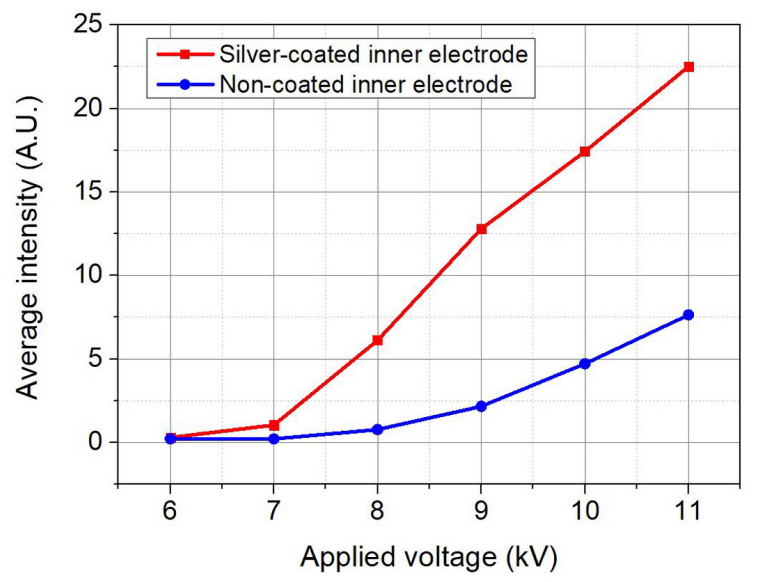

Figure 5. Average intensities according to applied voltages in the range of 6 to 11 $\mathrm{kV}$ and obtained by image processing of DBD images without any filters.

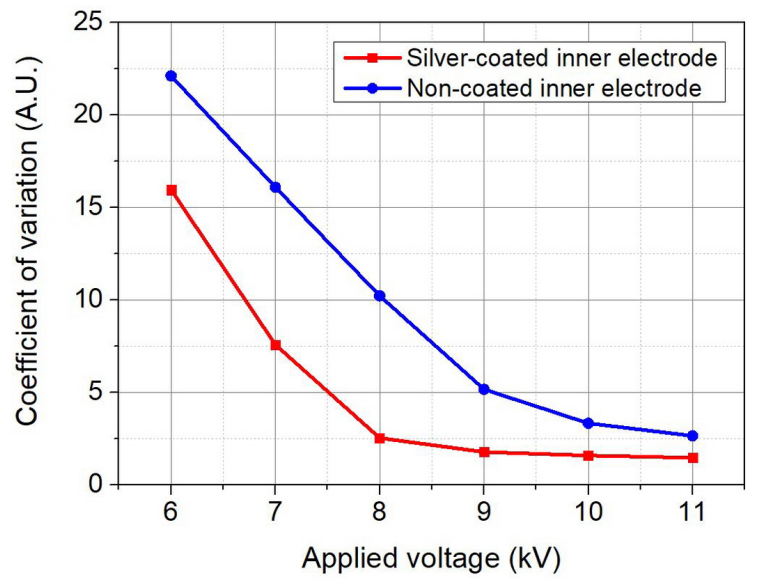

Figure 6. Coefficient of variation according to applied voltages in the range of 6 to $11 \mathrm{kV}$ and obtained by the image processing of DBD images without any filters.

(a)

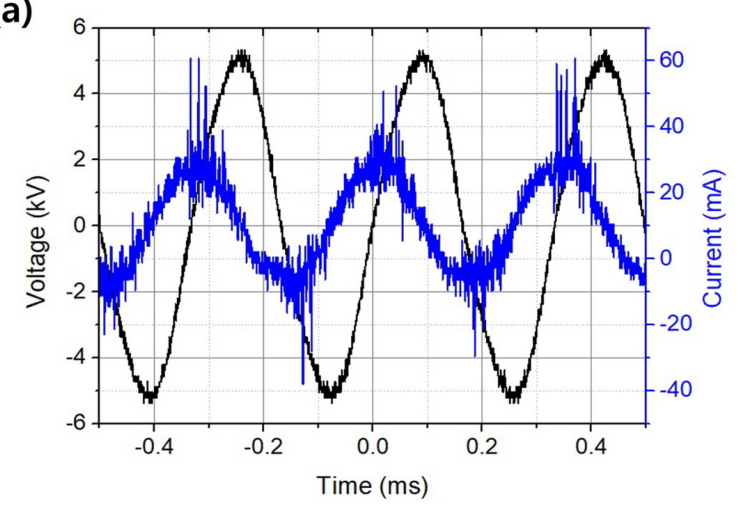

(b)

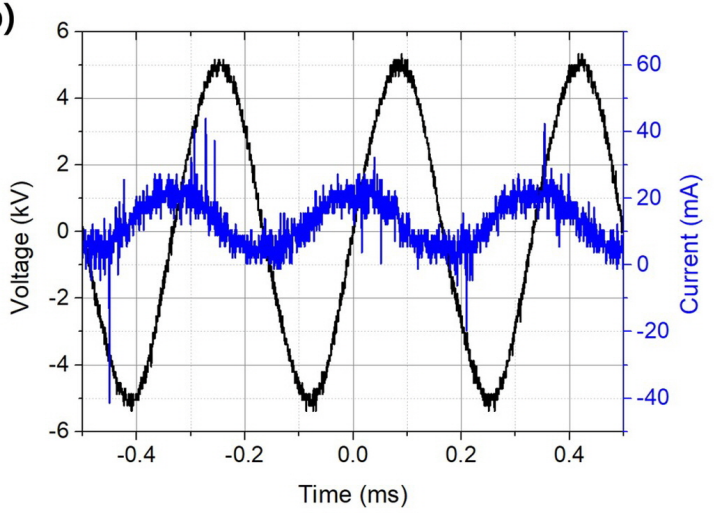

Figure 7. Voltage and current waveforms of the DBD device using (a) the silvercoated and (b) non-coated inner electrode at the applied voltage of $11 \mathrm{kV}$.

$\mathrm{kV}$ was numerically similar to that of the silver-coated inner electrode for an applied voltage of $8 \mathrm{kV}$. In particular, the average intensity of the silver-coated inner electrode was 7.98 times higher than that of the non-coated inner electrode at the applied voltage of $8 \mathrm{kV}$.

\section{Coefficient of variation}

The uniformities of the DBD was compared via the coefficient of variation $(\mathrm{CV})$ of the intensities in an image. The standard deviation is required to calculate the $\mathrm{CV}$ and is obtained as the measure of dispersion from the average of the histogram. The lower the standard deviation, the higher is the data tendency to approach the average of 

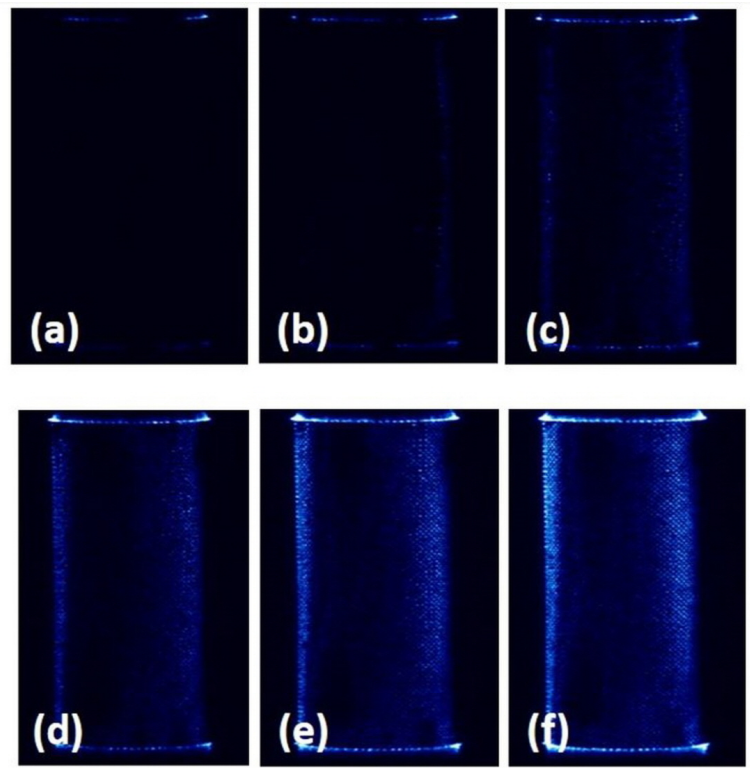

Figure 8. Band-pass filtered images of DBD by using the silver-coated inner electrode for applied voltages of (a) 6 , (b) 7 , (c) 8 (d) 9 (e) 10 , and (f) $11 \mathrm{kV}$ are observed with the ICCD camera.
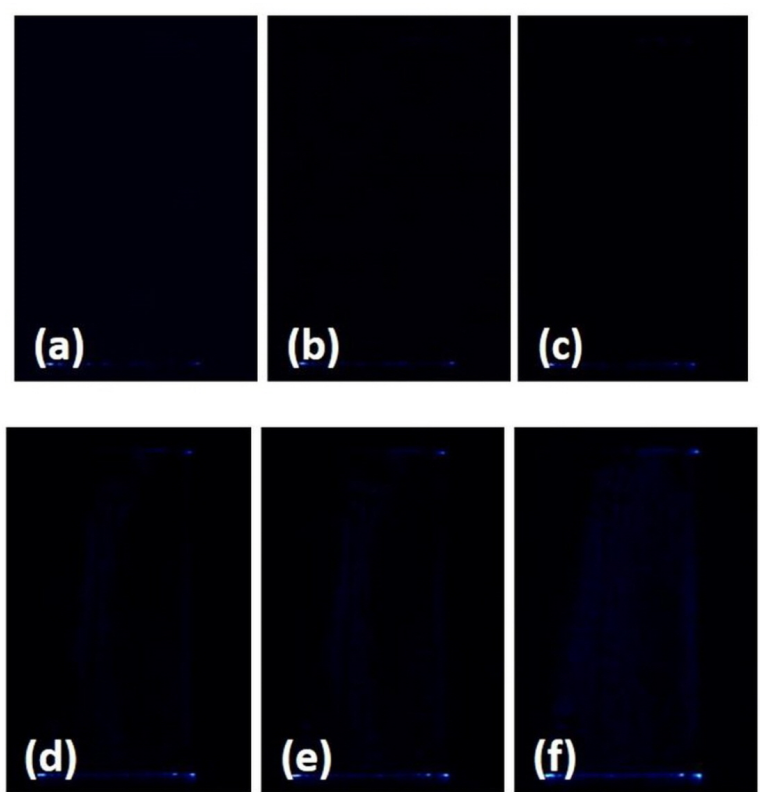

Figure 9. Band-pass filtered images of DBD by using the non-coated inner electrode for applied voltages of (a) 6, (b) 7, (c) 8, (d) 9, (e) 10, and (f) $11 \mathrm{kV}$ are observed with the ICCD camera.

the histogram. The standard deviation of the graylevel intensities of the histogram can be determined as follows:

$$
\begin{aligned}
s t d= & {\left[\frac{1}{(M \times N)(M \times N-1)}\right.} \\
& \left.\left((M \times N) \sum_{x=0}^{M-1} \sum_{y=0}^{N-1} g(x, y)^{2}-\left(\sum_{x=0}^{M-1} \sum_{y=0}^{N-1} g(x, y)\right)^{2}\right)\right]^{\frac{1}{2}}
\end{aligned}
$$

The CV is a normalized measure of the dispersion of $g(x, y)$ and is defined as the ratio of the standard deviation to the average graylevel

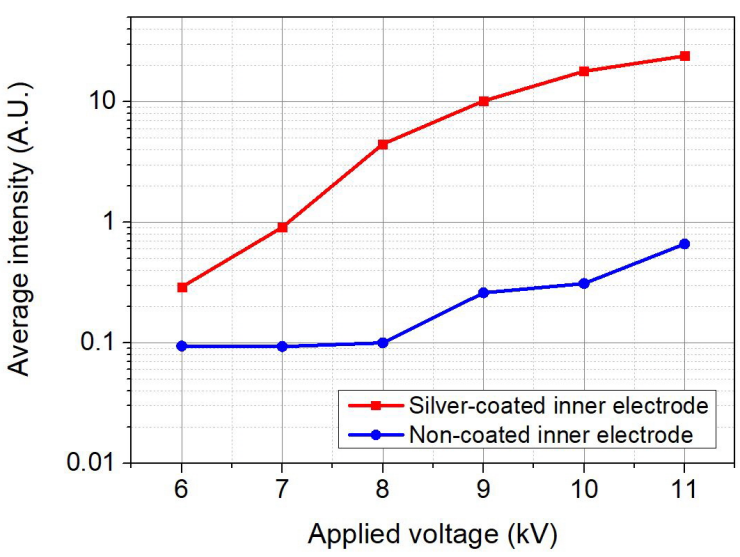

Figure 10. Average intensities of $\mathrm{OH}$ radical for applied voltages in the range of 6 to $11 \mathrm{kV}$ obtained by image processing of band-pass filtered images of DBD.

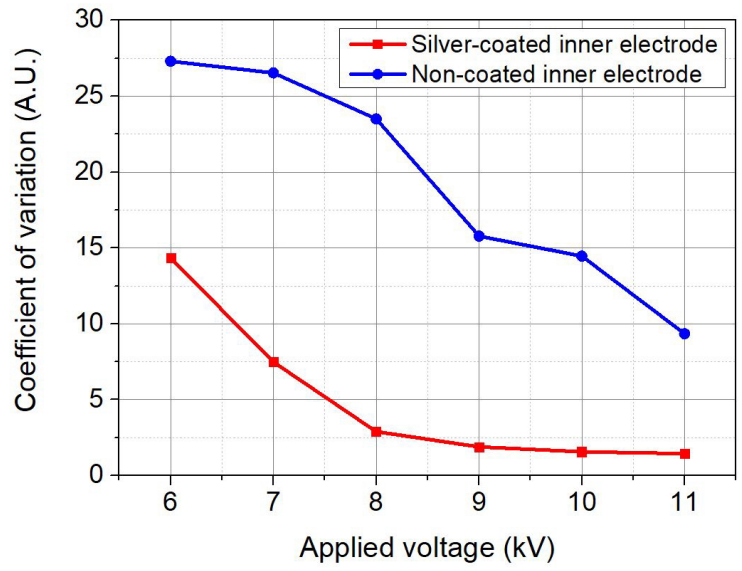

Figure 11. Coefficients of variation of $\mathrm{OH}$ radical for applied voltage in the range of 6 to $11 \mathrm{kV}$ obtained by image processing of band-pass filtered images of DBD.

value, as follows:

$$
C V=\frac{s t d}{\bar{g}}
$$

The CV can be used to compare the standard deviations for different average graylevel values. The lower the value of the $\mathrm{CV}$, the more uniform the DBD, and the higher the value of the $\mathrm{CV}$, the more nonuniform the DBD. Figure 6 shows that the silver-coated inner electrode produces more uniform results than the non-coated inner electrode for applied voltages in the range of 6 to $11 \mathrm{kV}$. In addition, the uniformity of the non-coated inner electrode at an applied voltage of $11 \mathrm{kV}$ was similar to that of the silver-coated inner electrode at an applied voltage of $8 \mathrm{kV}$. Moreover, the uniformity of the silver-coated inner electrode was $75 \%$ higher than that of the non-coated inner electrode at the applied voltage of $8 \mathrm{kV}$.

Figure 7 shows the voltage and current waveform of the DBD device using the silver-coated and non-coated inner electrode. The current amplitude and spike of the silver-coated inner electrode are higher than the non-coated inner electrode. The DBD device using the silvercoated inner electrode generated more discharge at the applied voltage of $11 \mathrm{kV}$. As a result, the intensity and uniformity of the silver-coated inner electrode are higher than for the non-coated inner electrode owing to the silver-coated inner electrode being manufactured without any air gaps between the dielectric and electrode. 


\subsection{Intensity and uniformity of $\mathrm{OH}$ radical}

The OH radical is a common type of ROS in such DBD reactions and is generally associated with bacterial inactivation. Figures 8 and 9 show the DBD images according to the voltages applied to the DBD device. The DBD images were obtained using a band-pass filter along with the ICCD camera. Visually speaking, when the applied voltage was fixed, the intensities of the silver-coated inner electrode was observed to be considerably improved compared to those of the noncoated inner electrode.

Figures 10 and 11 depict the average intensities and uniformities for the $\mathrm{OH}$ radical produced by the DBD process; note that because of the numerical differences, Fig. 10 is presented on a logarithmic scale. The $\mathrm{OH}$ radicals generated at the silver-coated inner electrode enhance the intensities and uniformities of $\mathrm{OH}$ radicals by up to 57.8 and 9.27 times, respectively, compared to the non-coated inner electrode. Sun et al. studied the $\mathrm{OH}$ radical by optical emission spectroscopy under atmospheric pressure, and they noted that the generation of $\mathrm{OH}$ radicals produced by corona discharge improved with smaller discharge gaps [13]. Thus, more $\mathrm{OH}$ radicals are generated in the presence of a stronger electric field. The silver-coated inner electrode generates a higher electric field because the distance between the dielectric and electrode is decreased; thus, the silver-coated inner electrode can produce more $\mathrm{OH}$ radicals than the non-coated inner electrode.

\section{Conclusions}

A silver-coated inner electrode was fabricated on the surface of a dielectric using the silver mirror reaction method to generate an enhanced DBD. Traditionally, non-coated inner electrodes have unnecessary air gaps between the dielectric and electrode. However, the silver-coated inner electrode could be manufactured without such air gaps. The intensity and uniformity of the DBD was compared for the coated and non-coated inner electrodes to investigate the effects of the air gap elimination. The DBD images were obtained using an ICCD camera for both the coated and non-coated inner electrodes and processed with the OpenCV library of Python. The characteristics of the silver-coated inner electrode were observed to be better than those of the non-coated inner electrode at a given voltage. Consequently, the silver-coated inner electrode has improved plasma intensities of up to 7.98 times and improved plasma uniformities of up to $75 \%$ compared to the non-coated inner electrode; the CV and average intensity of the non-coated inner electrode for an applied voltage of $11 \mathrm{kV}$ were similar to those of the silver-coated inner electrode for an applied voltage of $8 \mathrm{kV}$. Therefore, the silver-coated inner electrode without air gaps lowered the breakdown voltage of $3 \mathrm{kV}$ for generating the DBD. Moreover, the $\mathrm{OH}$ radicals produced by the $\mathrm{DBD}$ were compared using band-pass filtered data obtained using the ICCD camera. The $\mathrm{OH}$ radical produced at the silver-coated inner electrode showed higher intensities of up to 57.8 times and higher uniformities of up to 9.27 times compared to the non-coated inner electrode. Thus, we conclude that better DBD can be achieved when there are no air gaps between the electrode and dielectric, and our proposed silver mirror reaction method is suitable for eliminating air gaps.

\section{Acknowledgements}

This work was supported by the National Research Foundation of Korea(NRF) grant funded by the Korea government(MISP) (No.2019R1F1A1042084)

\section{References}

[1] H. Ayan, D. Staack, G. Fridman, A. Gutsol, Y. Mukhin, A. Starikovskii, A. Fridman, and G. Friedman, J. Phys. D Appl. Phys. 42, 125202 (2009).

[2] S. Müller and R. J. Zahn, Contrib. Plasma Phys. 47, 520 (2007).

[3] G. Fridman, M. Peddinghaus, M. Balasubramanian, H. Ayan, A. Fridman, A. Gutsol, and A. Brooks, Plasma Chem. Plasma Process. 26, 425 (2006).

[4] G. Borcia, C. A. Anderson, and N. M. D. Brown, Plasma Sources Sci. Technol. 12, 335 (2003).

[5] S. Y. Moon and A. Y. Moon, Appl. Sci. Converg. Technol. 28, 17 (2019).

[6] T. Shao, G. S. Sun, P. Yan, J. Wang, W. Q. Yuan, Y. H. Sun, and S. Zhang, J. Phys. D Appl. Phys. 39, 2192 (2006).

[7] Q. Ye, Y. Wu, X. Li, T. Chen, and G. Shao, Plasma Sources Sci. Technol. 21, 065008 (2012).

[8] Y. Ma, G. J. Zhang, X. M. Shi, G. M. Xu, and Y. Yang, IEEE Trans. Plasma Sci. 36, 1615 (2008).

[9] J. Choi and Y. W. Kim, Korean Intellectual Property Office, 102020-0064557, (2020).

[10] W. E. Benet, G. S. Lewis, L. Z. Yang, and D. E. P. Hughes, J Chem. Res. 35, 675 (2011).

[11] S. H. Hong, T. H. Kim and S. S. Choi, Appl. Sci. Converg. Technol. 28, 101 (2019).

[12] H. P. Dorn, R. Neuroth, and A. Hofzumahaus, J. Geophys. Res. 100, 7397 (1995).

[13] M. Sun, Y. Wu, J. Li, N. H. Wang, J. Wu, K. F. Shang, and J. L. Zhang, Plasma Chem. Plasma Process. 25, 31 (2005). 